\title{
Down-Regulation of IncRNA XIST Ameliorates Lipoprotein(a) Induced Endothelial Progenitor Cell Damage via Modulating miR-126/PLK2 Axis
}

Xiaolei Zhang ( $\nabla$ zzzzhangxiaolei@163.com )

Jiangsu College of nursing https://orcid.org/0000-0001-5977-0374

Guoying Xu

Jiangsu College of Nursing

Shizhen Wang

Jiangsu College of Nursing

Pei Chen

Jiangsu College of Nursing

Shanshan Chen

Jiangsu College of Nursing

Research

Keywords: endothelial progenitor cell, XIST, miR-126, PLK2, damage

Posted Date: September 13th, 2021

DOl: https://doi.org/10.21203/rs.3.rs-757604/v1

License: (c) (1) This work is licensed under a Creative Commons Attribution 4.0 International License.

Read Full License 


\section{Abstract \\ Background}

Recent studies have discovered that IncRNAs regulate lipoprotein (LP) (a)-mediated endothelial progenitor cells (EPCs) damage through sponging miRNAs. However, the role of XIST in EPC remains unknown.

\section{Method:}

Firstly, LP(a)(100 ng/mL) was used to treat EPCs for 6 hours. Then, the apoptosis, proliferation, migration, adhesion, angiogenesis of EPCs were detected. Moreover, overexpression of XIST or miR-126 on EPCs were built. Mechanistically, bioinformatics database, dual-luciferase reporter assay and RIP were used to found the binding relationships between XIST and miR-126, miR-126 and PLK2.

\section{Results}

Presently, we found LP(a) treatment significantly induced apoptosis, attenuated the proliferation, migration, adhesion, angiogenesis of EPCs. While knocking down XIST or overexpression miR-126 significantly reversed LP(a) induced damage on EPCs. Moreover, forced overexpression of XIST partially offset the protective effects of overexpressing miR-126 on EPC. Mechanistically, through bioinformatics database (http://starbase.sysu.edu.cn/index.php), we found potential binding relationships between XIST and miR-126, miR-126 and PLK2. Furthermore, the dual-luciferase reporter assay and RIP experiment confirmed the targeted binding between them.

\section{Conclusion}

Collectively, the above results confirmed that down-regulating XIST improved LP(a)-induced EPC damage by regulating the miR-126/PLK2 axis, and the XIST/miR-126/PLK2 axis exerted an essential role in regulating EPC function.

\section{Highlights}

1. Knocking down XIST reduced apoptosis and enhanced proliferation of EPCs.

2. knocking down XIST promoted the migration and angiogenesis of EPC cells

3. XIST sponged miR-126

4. XIST upregulated PLK2 via targeting miR-126.

\section{Introduction}


Endothelial progenitor cells (EPCs) were discovered and named by Asahara et al. in 1997 as the precursor cells of mature ECs. In fact, EPCs are a group of cells with complete differentiation grade and a collection of progenitors at different differentiation stages, with heterogeneity ${ }^{1-2}$. Currently, it is believed that EPCs, etc. express hematopoietic progenitor cell markers CD34 and CD133, and have specific homing to injured or ischemic sites, being involved in angiogenesis and re-endothelialization. Recent studies have shown that angiogenesis is an important compensatory response to chronic ischemic disease ${ }^{3}$. The number and function of EPCs in the peripheral blood circulation determine the extent of endothelial repair of damaged vessels. Thus, the number of circulating EPCs in patients with AS and diabetes is reduced, and EPC function is impaired ${ }^{4-5}$. Studies have demonstrated that ox-LDL can further induce EPC apoptosis by increasing oxidative stress and inflammatory response, thus weakening its vascular repair ability ${ }^{6}$. Therefore, elucidating the molecular mechanism of ox-LDL-mediated EPCs is of great value in exploring the occurrence and development of AS and developing effective therapeutic drugs for AS.

Recent studies have discovered that IncRNAs regulate lipoprotein (LP)(a)-mediated EPC damage through sponging miRNAs ${ }^{7}$. For example, IncRNA GUSBP5-AS is up-regulated in EPCs in patients with deep vein thrombosis (DVT). LncRNA GUSBP5-AS regulates the expression of FGF2 and MMP2/9 by targeting the miR-223-3p/FOX01/Akt pathway, thus modulating the angiogenesis, proliferation and homing capacity of EPCs ${ }^{8}$. In contrast, Yu Guo et al. reported that IncRNA TUG1 exerts an essential role in regulating migration and differentiation of EPCs. LncRNA TUG1 serves as the competitive endogenous RNA of miR6321, and miR-6321 inhibits EPC migration and differentiation via its target ATF2 ${ }^{9}$. In addition, IncRNA GAS $5^{10}$, IncRNA-MALAT $1^{11}$, IncRNA-AK $131850^{12}$ and other IncRNAs affect the expression of miRNAs to contribute to regulating EPC functions. LncRNA XIST, as a common oncogenic non-coding RNA, plays an oncogenic role in non-small cell lung cancer (NSCLC) ${ }^{13}$, chordoma ${ }^{14}$, osteosarcoma ${ }^{15}$ and other tumors. Besides, XIST gene knockout increases the activity of IL-1 $\beta$-treated chondrocytes and attenuates apoptosis and extracellular matrix protein degradation in osteoarthritis (OA). What's more, XIST gene knockout inhibits the occurrence and development of OA through the miR-149-5P/DNMT3A axis ${ }^{16}$. Thus, the XIST gene knockout alleviates inflammatory response and cell damage. However, the role of IncRNA$\mathrm{XIST}$ in regulating EPCs remains elusive.

MiR-126, as a common miRNA, has been reported in multiple tumor literature. For instance, Li M et al. believed that the miR-126 expression is enhanced in esophageal squamous cell carcinoma (ESCC) cells and tissues. Down-regulation of miR-126 reduces ESCC cell survival and promotes its death by regulating autophagic proteins LC3B and P6 $2^{17}$. Nevertheless, studies in bladder cancer have revealed that the expression of miR-126 is increased, which is closely related to tumor grade ${ }^{18}$. Therefore, the role of miR126 in tumors is diversified, and it may play different roles in different tumors. More importantly, relevant studies have also shown miR-126 plays a role in regulating EC function. For example, Zhang Lin et al. reported that miR-126 is down-regulated in OGD-induced HBMECs, and overexpressing miR-126 activates the PI3K/Akt pathway, thereby affecting the proliferation and apoptosis of HBMECs ${ }^{19}$. Hence, it can be speculated that miR-126may also contribute to regulating EC function. 
Additionally, PLK2, belongs to the Polo-like kinase (PLK) family, mainly contributes to regulating cell cycle or mitosis ${ }^{20}$. PLK2 is known to exert a vital function in inhibiting cancer progression by regulating centriolar replication and being activated by spindle checkups p53-dependently. The study of Liu Li Ying et al. in gastric cancer confirmed that the tumor suppressor gene PLK2 is directly modulated by miR-126 in SGC-7901 cells. MiR-126 overexpression not only abates SGC-7901 cell growth and colony formation, but also induces apoptosis in vitro, while miR-126 does not affect the cell cycle of gastric cancer ${ }^{21}$. Thus, miR-126 targets PLK2 to regulate the proliferation of cancer cells. More importantly, Chang Lili et al. reported that miR-126 overexpression enhances cell proliferation and migration, while dampening miR126 has the reverse effect. This study found that PLK2 was the target gene of miR-126 and could inhibit keratinocytes' survival, colony formation and migration ${ }^{22}$. However, few studies have reported that miR126 overexpression does not decrease the PLK2 level in NSCLC cells, which may be closely related to the cell-type-specific inhibition of specific miRNAs on target genes ${ }^{23}$. However, the role and specific mechanism of PLK2 in regulating EPC function are still unclear.

Overall, this study speculated that down-regulating XIST improved LP(a)-induced EPC damage by regulating the miR-126/PLK2 axis, and the XIST/miR-126/PLK2 axis exerted an essential role in regulating EPC function.

\section{Materials And Methods}

\subsection{Isolation, culture and identification of EPCs}

Human umbilical cord blood was collected, and mononuclear cells were isolated by density gradient centrifugation. The cells were inoculated $\left(4 \times 10^{6}\right)$ and cultured in EC medium (ECM) for 4 days, and the non-adherent cells were washed away with PBS. Then, the medium was altered, and the cells were cultured until 7 days. Subsequently, the cells were inoculated into 6-well plates, and the supernatant was removed. The expression of CD34 + and CD133 + was determined by flow cytometry.

\subsection{Transfection and treatment of XIST and miR-126}

After trypsinization, EPCs were re-suspended and the cell density was adjusted to $5 \times 10^{7} \mathrm{cells} / \mathrm{mL}$. After preheating with the electrophoresis apparatus for $15 \mathrm{~min}, 100 \mu \mathrm{L}$ EPCs were taken and added into the EP tube. Then, XIST knockdown sequences (si-XIST\#1, si-XIST\#2), XIST overexpression sequence (XIST), miR-126 mimics or inhibitors or negative controls were supplemented and mixed. Afterward, the cells and the mixture were gently transferred to the rotor and electroporated (180 V, $25 \mathrm{~ms})$ after pre-cooling on ice for $10 \mathrm{~min}$. After the electroporation was completed, the rotor was removed and balanced at room temperature (RT) for $10 \mathrm{~min}$. After the transfected cells were cultured for 24 hours, the transfection validity of miR-126 was verified by reverse transcription-polymerase chain reaction (RT-PCR). EPCs were treated with $100 \mathrm{ng} / \mathrm{mL}$ ox-LDL for 6 hours to simulate cell injury.

\subsection{Proliferation analysis of EPCs}


MTT was conducted to calculate the number of cell proliferation. EPCs proliferated in vitro were trypsinized with $0.25 \%$ trypsin and counted. Then, $200 \mu \mathrm{L}$ EPCs $\left(1 \times 10^{7}-1 \times 10^{8} / \mathrm{L}\right)$ were uniformly inoculated onto 96 -well gelatin-coated plates and cultured for 28 hours. Subsequently, each well was supplemented with $10 \mu \mathrm{L} \mathrm{MTT}$, and the plates were further incubated at $37{ }^{\circ} \mathrm{C}$ with $5 \% \mathrm{CO}_{2}$ for 4 hours. Afterward, the supernatant was discarded, and dimethyl sulfoxide was added ( $150 \mu \mathrm{L} /$ well). Finally, the plates were oscillated for 5 min with an oscillator, and placed in a microplate reader to examine the optical density (OD) at the wavelengths of $490 \mathrm{~nm}$.

\subsection{EPC apoptosis}

Apoptosis was examined with the annexin V-FITC/PI staining kit (BD Biosciences, New Jersey, USA). Stably transfected cells were washed with cold PBS, re-suspended in binding buffer $(100 \mathrm{mmol} / \mathrm{L} \mathrm{NaCl}$, $25 \mathrm{mmol} / \mathrm{L} \mathrm{CaCl}_{2}, 100 \mathrm{mmol} / \mathrm{L}$ HEPES, pH7.4) and stained with V-FITC/PI at RT for $15 \mathrm{~min}$ in the dark. Finally, FACS flow cytometry (BD FACS Calibur, Becton, Dickinson and Company Biosciences, San Jose, USA) was employed to assess the apoptosis rate. Each experiment was repeated three times.

\subsection{EPC migration}

The migration of EPCs was tested by Boyden transwell assay. First, EPCs were trypsinized with trypsin/EDTA. Then, EPCs were seeded in the upper chamber of 24-well plates with polycarbonate membranes $\left(4 \times 10^{4} /\right.$ well) and cultured in the serum-free endothelial growth medium, while the medium containing VEGF $(50 \mathrm{ng} / \mathrm{mL})$ was placed in the lower chamber. After 24 hours of culture, the membranes were washed with PBS, and then fixed with $4 \%$ paraformaldehyde. The upper side of the membranes was gently wiped with cotton balls, and the membranes were then stained with hematoxylin. The migration of EPCs was calculated by counting cells in each well under the microscope.

\subsection{EPC adhesion assay}

The in vitro proliferated EPCs were trypsinized and counted. Then, $2 \mathrm{~mL}$ suspension of EPCs $\left(1 \times 10^{7} / \mathrm{L}\right)$ was inoculated in six-well plates and incubated for 48 hours. The same number of EPCs were trypsinized and inoculated to the gelatin-coated cover glass, and then incubated at $37^{\circ} \mathrm{C}$ with $5 \% \mathrm{CO}_{2}$ for $30 \mathrm{~min}$. The non-adherent cells were washed with PBS, and the adherent cells were counted at random from 3 microscope fields (20x), and the mean number was obtained.

\subsection{Detection of EPC colony formation}

The cells that were not adherent within 48 hours were inoculated in hybridoma dishes at a density of $10^{7} / \mathrm{mL}$. After further culture for a week, EPC-CFUs with typical morphology in each hybridoma dish were counted under a microscope by two people. Namely, a cluster of cells was centered around, and free round cells, spindle cells and needle-like cells around were chosen. Each group was counted twice and the mean was taken. The primary EPC-CFUs from the bone marrow of each mouse was treated with $0.25 \%$ trypsin and dispersed, and then inoculated in gelatin-coated six-well plates with $10^{3}-10^{4} / \mathrm{mL}$. The number 
of EPC-CFUs (9 wells/group) within a week was counted, and the colony unit size and cell density were observed.

\subsection{In vitro angiogenesis analysis}

In vitro angiogenesis kit was used to test the angiogenesis of EPCs. The ECMatrixTM glue solution and ECM 10x diluent was placed in a refrigerator at $4{ }^{\circ} \mathrm{C}$ overnight for freezing-thawing. Then, $100 \mu \mathrm{L} E C M$ 10x diluent was added to every $900 \mu \mathrm{L}$ ECMatrixTM solution and mixed. Afterward, the mixture was added to 96 -well plates $(50 \mu \mathrm{L} /$ well $)$ and incubated at $37^{\circ} \mathrm{C}$ for 1 hour for gelatinization. EPCs were obtained by trypsinizing the adherent cells, and then they were re-suspended in the culture medium with the cell density adjusted to $5 \times 10^{4} / \mathrm{mL}$. Subsequently, the EPCs were inoculated into ECMatrixTM glue (5000 10000/well). The canaliculization (angiogenesis) was observed under a 200-fold inverted microscope after 24 hours of culture at $37^{\circ} \mathrm{C}$. Canaliculization was recognized when the cells were elongated and deformed to a length of more than 4 times the width. The total length of the vasculature was measured and calculated.

\subsection{Dual-luciferase reporter assay}

Luciferase report vectors (XIST-WT/XIST-MT; PLK2-WT/PLK2-MT) were constructed by GenePharma (Shanghai, China). EPCs were seeded in 48-well plates and cultured to $70 \%$ confluence. Then, XISTWT/XIST-MT and PLK2-WT/PLK2-MT were co-transfected with miR-126 mimics of negative controls (miR-NC) into EPCs according to the Lipofectamine 2000 Reagent (Invitrogen, Carlsbad, CA, USA). Fortyeight hours after transfection, the luciferase activity was validated as per the manufacturer's instructions. All experiments were done in triplicate.

\subsection{RNA immunoprecipitation (RIP)}

First, $2 \times 10^{7}$ MPCs were collected, and the RIP lysates of the same volume were added. Then, the cells were centrifuged (12,000 rpm, with a diameter of $420 \mathrm{~mm}, 10 \mathrm{~min})$, and the supernatant was obtained. Next, $900 \mu \mathrm{L}$ RIP buffer (including RNase inhibitor, protease inhibitor, DNase) and $100 \mu \mathrm{L}$ cell lysate was added to the EP tube containing magnetic beads, and the IgG antibodies or Ago2 antibodies were supplemented for incubation at $4^{\circ} \mathrm{C}$ overnight. After that, the cells were centrifuged $(12,000 \mathrm{rpm}$, with a diameter of $420 \mathrm{~mm}, 10 \mathrm{~min}$ ), and the supernatant was removed. After washing with $500 \mu \mathrm{L}$ RIP wash buffer for 6 times, RNA purification was carried out immediately. Finally, the purified RNA was dissolved in $15 \mu \mathrm{L}$ DEPC water for quantitative RT-PCR.

\subsection{RT-PCR}

The total RNA in brain tissues and cells was extracted with Trizol (Tiangen, China) according to the instructions. After the RNA purity and concentration were determined, RevertAid First Strand cDNA Synthesis Kit (Thermo Scientific, Waltham, MA, USA) was adopted for reverse transcription. The specific amplification primers of quantitative PCR were shown in Table 1. U6 was used as internal reference of miR-126, and GAPDH was for internal reference of XIST and PLK2. PCR was conducted according to the 
guidelines of the SYBRß Premix Ex Taqand kit (Takara Biotech Corporation, Dalian, China) with Bio-Rad CFX96 (Bio-Rad, USA). Each sample was tested three times, and the results obtained were analyzed with the $2^{-\triangle \Delta C T}$ method.

Table 1

PCR primer sequences

\begin{tabular}{|c|c|}
\hline Name & Primer sequence $\left(5^{\prime}-3^{\prime}\right)$ \\
\hline \multirow[t]{2}{*}{ XIST } & Forward, CCATTGAAGATACCACGCTGC \\
\hline & Reverse, GGTTGTTGCCCAGGGTAGTG \\
\hline \multirow[t]{2}{*}{ miR-126 } & Forward, GGGGTCGTACCGTGAGT \\
\hline & Reverse, CAGTGCGTGTCGTGGAGT \\
\hline \multirow[t]{2}{*}{ PLK2 } & Forward, CTTCACTCCGGACAGACTGT \\
\hline & Reverse, GCTCCTCATCTGTCCTGTGT \\
\hline \multirow[t]{2}{*}{ U6 } & Forward, GCTTCGGCAGCACATATACTAAAAT \\
\hline & Reverse, CGCTTCACGAATTTGCGTGTCAT \\
\hline \multirow[t]{2}{*}{ GAPDH } & Forward, TGAAGGGTGGAGCCAAAAG \\
\hline & Reverse, AGTCTTCTGGGTGGCAGTGAT \\
\hline
\end{tabular}

\subsection{Statistical analysis}

The data were expressed as mean \pm standard deviation, statistical software Prism GraphPad 7.0 (GraphPadSoftware, USA) was adopted for statistical analysis, and differences between groups were compared by one-way ANOVA or $t$ test. $P<0.05$ indicated significant difference.

\section{Results}

\subsection{Knocking down IncRNA XIST significantly reduced the injury of LP(a)-mediated EPCs}

PCR results confirmed that the XIST expression was distinctly up-regulated after EPCs were treated with $100 \mathrm{ng} / \mathrm{mL} \mathrm{LP}(\mathrm{a})$ for 6 hours (Fig. 1A). Apoptosis was examined by flow cytometry and cell proliferation was detected by MTT. The results confirmed that after LP(a) treatment, EPC apoptosis was increased and the cell proliferation was inhibited (Fig. 1B-C). More importantly, knocking down XIST significantly reduced apoptosis and enhanced proliferation of EPCs (Fig. 1B-C). In addition, the results of migration, adhesion assay and colony formation experiment showed that the migration, adhesion and colony formation of EPC cells were attenuated after LP(a) treatment, while knocking down XIST exerted the 
reversed effects (Fig. 1D-F). Moreover, the angiogenesis experiment manifested that after LP(a) treatment, the angiogenesis of EPCs decreased, while knocking down XIST facilitated the angiogenesis (Fig. 1G). The above results demonstrated that knocking down IncRNA XIST notably reduced the injury of the LP(a)-mediated EPCs.

\subsection{Overexpressing miR-126 mitigated the injury of LP(a)- mediated EPCs}

EPCs overexpressing miR-126were induced (Fig. 2A), and EPCs were treated with LP(a) at a dose of 100 $\mathrm{ng} / \mathrm{mL}$ for 6 hours. Then, EPC apoptosis was verified by flow cytometry, and cell proliferation was determined by MTT. As a result, overexpressing miR-126 dampened apoptosis and enhanced proliferation of EPCs (Fig. 2B-C). Besides, the results of migration assay, adhesion assay and colony formation experiment indicated that the migration, adhesion and colony formation of EPCs were all weakened after $\mathrm{LP}(\mathrm{a})$ treatment, while they were all elevated after overexpressing miR-126 (Fig. 2D-F). Furthermore, the angiogenesis experiment proved that the angiogenesis of EPCs decreased after $\operatorname{LP}(\mathrm{a})$ treatment, while overexpressing miR-126 notably improved EPC angiogenesis (Fig. 2G). These results demonstrated that overexpressing miR-126mitigated the injury of LP(a)-treated EPCs.

\subsection{LncRNA XIST targeted miR-126}

Moreover, we searched the targeted gene of XIST on the bioinformatics database Starbase (http://starbase.sysu.edu.cn/index.php) to further clarify the association between XIST and miR-126, and discovered that XIST targeted miR-126 (Fig. 3A). Furthermore, the dual-luciferase reporter assay and RIP experiment were carried out to determine the targeted binding between XIST and miR-126. Interestingly, miR-126 mimics weakened the luciferase activity of XIST-WT, while had no effect on that of XIST-MUT ( $P$ $<0.05$, Fig. 3B). Meanwhile, RIP manifested that XIST was up-regulated in Ago2 after supplementing with miR-126 mimics $(P<0.05$, Fig. $3 C$ ). Meanwhile, miR-126 was attenuated in $L P(a)$ treatment, and was enhanced in EPCs with XIST overexpression plasmids (Fig. 3D). The above results suggested that XIST targeted miR-126.

\subsection{IncRNA XIST targeted miR-126 to regulate LP(a)- mediated EPC damage}

XIST overexpression plasmids were supplemented in EPCs overexpressing miR-126 for investigating whether XIST in exosomes targeted miR-126 to regulate EPC function $(P<0.05$, Fig. 4A). The results of apoptosis and proliferation assay testified that overexpressing miR-126 reduced the apoptosis, and promoted the proliferation of EPCs. However, XIST supplementation on this basis partially offset the regulatory effects of overexpressing miR-126 on EPC proliferation and apoptosis (Fig. 4B-C). Moreover, the results of migration, adhesion and colony formation experiments confirmed that overexpressing miR126 improved the migration, adhesion and colony formation of EPCs treated with LP(a), while 
supplementation of XIST on the basis of miR-126 overexpression abated the migration, adhesion and colony formation of EPCs (Fig. 4D-F). Furthermore, the angiogenesis experiment proved that supplementing XIST repressed the angiogenesis capacity of EPCs (Fig. 4G). These findings testified that XIST targeted miR-126, which regulated the EPC damage mediated by LP(a).

\section{5 miR-126 targeted PLK2}

Besides, we searched the targeted gene of miR-126 with the Starbase to further clarify the association between PLK2 and miR-126, and found that miR-126 targeted PLK2 (Fig. 5A). Furthermore, dualluciferase reporter assay and RIP were implemented to clarify the targeted relationship between PLK2 and miR-126. Interestingly, miR-126 mimics markedly dampened the luciferase activity of PLK2-WT, while had no effect on that of PLK2-MUT ( $P<0.05$, Fig. 5B). RIP experiment proved that PLK2 expression was facilitated in Ago2 after supplementing miR-126 mimics $(P<0.05$, Fig. $5 C)$. These results confirmed that miR-126 targeted PLK2.

\section{6 miR-126 modulated the LP(a)-mediated EPC injury via targeting PLK2}

Further clarify whether miR-126 in exosomes targeted PLK2 and regulated EPC function. The results showed that miR-126 mimics attenuated PLK2 expression $(P<0.05$, Fig. 6A). Meanwhile, the detection of cell apoptosis and proliferation revealed that overexpressing PLK2 notably increased the apoptosis and abated the proliferation of EPCs. Nevertheless, the supplementation of miR-126 mimics on this basis partially offset the regulatory effects of overexpressing PLK2 on EPC proliferation and apoptosis (Fig. 6BC). In addition, the cell migration, adhesion and colony formation experiments confirmed that overexpressing PLK2 further reduced the LP(a)-treated EPC migration, adhesion and colony formation, which were all strengthened after supplementing miR-126mimics on the basis of overexpressing PLK2 (Fig. 6D-F). Furthermore, angiogenesis experiment proved that overexpressing PLK2 further declined the EPC angiogenesis, which was enhanced by miR-126mimics (Fig. 6G). These results demonstrated that miR-126 targeted PLK2 and modulated the injury of LP(a)-treated EPCs.

\section{Discussion}

AS is a complex chronic disease and an important cause of various secondary diseases 1-2. LP(a) is considered to be a major risk for the occurrence and development of AS by promoting foam cell formation, EPC dysfunction, plaque formation, and inflammatory response ${ }^{24-25}$. Therefore, LP(a) was transfected in EPCs in this study to simulate EPC damage in vitro. The results indicated that XIST targets the miR-126-3p/PLK2 axis to regulate the LP(a)-mediated EPC damage.

XIST, as a common IncRNA, has been extensively studied in a variety of diseases. XIST was initially discovered to silence $X$ chromosome genes via cis-acting ${ }^{26}$. Recent studies have suggested that the abnormal expression of XIST and its "ceRNA" effect as a miRNA sponge are responsible for the dysfunctions of many miRs, and plays a regulatory role in various neoplasms. Chen Zhenzhang et al. 
reported that XIST is highly expressed in esophageal cancer, and inhibiting XIST significantly attenuates the proliferation, migration and invasion of TE-1 and SKGT-4 cells, and induces their apoptosis. The abnormal expression of XIST may be involved in the occurrence and development of esophageal cancer through regulating the JAK2/STAT3 signaling pathway and miR-494/CDK6 axis ${ }^{27}$. Meanwhile, the carcinogenic effect of XIST has been demonstrated in thyroid cancer ${ }^{28}$, colorectal cancer ${ }^{29-30}$, laryngeal squamous cell carcinoma and other tumors ${ }^{31-34}$. In recent years, reports on XIST in other non-tumor diseases are also increasing. Zhou Tao et al. showed that XIST promotes myocardial infarction by targeting miR-130A-3p ${ }^{35}$. Consistent with the results reported by Zhou Tao et al., Yang Xiaoyu et al. found that XIST expression in cartilage specimens of OA patients is distinctly up-regulated, which confirms that XIST regulates the proliferation and apoptosis of OA chondrocytes through the miR-211/CXCR4 axis ${ }^{36}$. Studies in spinal cord injury have revealed that XIST gene knockout limited the neuronal apoptosis in rats, and its protective effect is regulated by the phosphorylation of AKT through competitive binding to miR$494^{37}$. Thus, it can be seen that XIST could strengthen cell apoptosis in inflammatory diseases, and knocking down XIST plays a protective role in injury. Above all, the role of XIST in EPC is scarcely reported. This study clarified that knocking down XIST significantly reduces LP(a)-induced EPC damage.

As a classical miR, miR-126 has been widely studied. Sabo Alexandru A et al. found that miR-126 is upregulated in plasma EVS and urine, and is positively correlated with tumor grade ${ }^{38}$. Studies in gastric cancer also have confirmed that miR-126 inhibits cell apoptosis and promotes its proliferation by downregulating the PI3K pathway, such as IGF1R, INSR, PDK1 and AKT1 ${ }^{39}$. Meanwhile, the carcinogenic effect of miR-126 has been proved in glioblastoma ${ }^{40}, \mathrm{NSCLC}^{41}$, porcine granulosa cells ${ }^{42}$, and other tumors. There are vast studies on miR-126 in AS, and most scholars believe that miR-126 plays a protective role in ECs. For instance, Ohta Momoka et al. hold that IL- 6 reduces the accumulation of miR-126 in EA.hy 926 cells, thus increasing the expression of miR-126-targeted genes. Moreover, IL- 6 enhances the adhesion of human mononuclear cell line THP-1, accompanied by an increase of intercellular adhesion molecule-1 level ${ }^{43}$. Xue Wen-long et al. confirmed that high glucose reduces the endogenous miR-126 level in HUVECs and increases the expression of DNMT1, thus resulting in the dysfunction of HUVEC migration 44 . In addition, studies in cerebral ischemia have found that overexpression of miR-126 dampens the expression of pro-inflammatory cytokines and adhesion molecules after ischemic stroke, thus alleviating the destruction of blood-brain barrier ${ }^{45}$. Furthermore, miR-126 mimics down-regulate VCAM- 1 in the bleeding area and ease the destruction of the blood-brain barrier after cerebral hemorrhage ${ }^{46}$. Thus, studies in both hemorrhagic/ischemic stroke and HUVECs show that miR-126 plays a positive role in protecting the function of HUVECs. The results of this study initially demonstrated the protective effect of miR-126 in LP(a)-mediated EPC damage.

Also, this study confirmed that miR-126 is negatively regulated by XIST. Although there have been vast studies on XIST, only Cheng Zhihua et al. reported that XIST regulates the IRS1/PI3K/Akt pathway as the ceRNA of miR-126 in glioma ${ }^{47}$. Consistent with previous reports, this study also proved that XIST gene knockout inhibits the survival, migration, invasion, anti-apoptosis and glucose metabolism of 
glioblastoma cells. Therefore, this study initially testified through compensation experiments in nontumor diseases that XIST sponges miR-126 as a ceRNA, thus regulating the function of EPC, which provides a basis for in-depth exploration of the role and mechanism of XIST in EPCs.

By further exploring the downstream mechanism of the XIST/miR-126 axis, we discovered PLK2 is the target of miR-126. PLK2 also was a cell cycle regulation gene that affects cell cycle progression, cell proliferation and individual bone development ${ }^{48}$. Meanwhile, PLK2 plays a carcinogenic role in multiple tumors, such as glioma ${ }^{49}$, oral cancer ${ }^{50}$ and colorectal carcinomas ${ }^{51}$. In contrast, down-regulating PLK2 inhibits synovial damage in KOA rats, promotes synovial cell apoptosis, dampens angiogenesis, cartilage damage, chondrocyte apoptosis and alleviates the inflammatory damage of synovial tissue and cartilage tissue in inflammatory diseases. PLK2 overexpression reverses the effects of miR-27a up-regulation on synovial angiogenesis and chondrocyte injury in KOA rats ${ }^{52}$. Ischemia/reperfusion (IR) up-regulates PLK2, but decreases the expression of miR-128. This study proved that PLK2 regulated by miR-128 induces apoptosis/death by activating the nuclear factor KB (NF-KB). MiR-128 and PLK2 are novel targets for the prevention of myocardial I/R or oxidative stress-mediated injury ${ }^{53}$. These studies have confirmed that PLK2 is significantly up-regulated in injuries. The findings of this study were consistent with the previous researches. Namely, PLK2 is significantly up-regulated in damaged EPCs, and overexpressing PLK2 further aggravates the damage of EPC, while compensation of miR-126 mimics alleviates the damage of EPC.

In conclusion, our study testified that knocking down XIST ameliorates LP(a)-induced EPC damage. The specific mechanism is that XIST sponges miR-126 as a ceRNA to regulate the expression of PLK2, which provides a new way to explore the treatment of AS.

\section{Declarations}

\section{Authors contribution}

Conceived and designed the experiments: Xiaolei Zhang,

Performed the experiments: Xiaolei Zhang, Guoying Xu, Shizhen Wang,

Statistical analysis: Pei Chen, Shanshan Chen,

Wrote the paper: Xiaolei Zhang.

All authors read and approved the final manuscript.

\section{Consent for publication}

Not applicable.

\section{Competing interests}


The authors declare that they have no competing interests.

\section{Ethics statement}

Our study was approved by the Ethics Review Board of Jiangsu College of Nursing.

\section{Data Availability Statement}

The data sets used and analyzed during the current study are available from the corresponding author on reasonable request.

\section{Acknowledgements}

Not applicable.

\section{Funding}

This work was supported by Huaian Natural Science Research Plan (No. HAB201844).

\section{References}

1. Wang Qian-Nan,Zou Zheng-Xing,Wang Xiao-Peng et al. Endothelial Progenitor Cells Induce Angiogenesis: a Potential Mechanism Underlying Neovascularization in Encephaloduroarteriosynangiosis.[J] .TransI Stroke Res, 2020, DOI囚10.1007/s12975-020-00834-9.

2. Rodríguez Cristina,Slevin Mark,Rodríguez-Calvo Ricardo et al. Modulation of endothelium and endothelial progenitor cell function by low-density lipoproteins: implication for vascular repair, angiogenesis and vasculogenesis.[J] .Pathobiology, 2009, 76: 11-22.

3. Nakamura Kouki,Taniguchi Takashi,Hirabayashi Megumi et al. Altered properties of endothelial cells and mesenchymal stem cells underlying the development of scleroderma-like vasculopathy in Klf5 ,Fli1 mice.[J].2020,DOIه10.1002/art.41423.

4. Yang Yong,Zhou Yong,Wang Yiyong et al. Exendin-4 reverses high glucose-induced endothelial progenitor cell dysfunction via SDF-1 $\beta /$ CXCR7-AMPK/p38-MAPK/IL-6 axis.[J] .Acta Diabetol, 2020, DOIه10.1007/s00592-020-01551-3.

5. Yu Tianlong,Jing Miao,Gao Yunyan et al. Study on the relationship between hyperthyroidism and vascular endothelial cell damage.[J] .Sci Rep, 2020, 10: 6992.

6. Qian Weibin,Cai Xinrui,Qian Qiuhai et al. Astragaloside IV protects endothelial progenitor cells from the damage of ox-LDL via the LOX-1/NLRP3 inflammasome pathway.[J] .Drug Des Devel Ther, 2019, 13: 2579-2589.

7. Chen Peier,Zhong Jianfeng,Ye Jianfeng et al. miR-324-5p protects against oxidative stress-induced endothelial progenitor cell injury by targeting Mtfr1.[J] .J. Cell. Physiol., 2019, 234: 22082-22092.

8. Sun Li-Li,Lei Feng-Rui,Jiang Xu-Dong et al. LncRNA GUSBP5-AS promotes EPC migration and angiogenesis and deep vein thrombosis resolution by regulating FGF2 and MMP2/9 through the 
miR-223-3p/FOXO1/Akt pathway.[J] .Aging (Albany NY), 2020, 12: 4506-4526.

9. Yu Guo,Li Sichen,Liu Peixi et al. LncRNA TUG1 functions as a ceRNA for miR-6321 to promote endothelial progenitor cell migration and differentiation.[J] .Exp. Cell Res., 2020, 388: 111839.

10. Yao Jiamei,Shi Zanhua,Ma Xinhua et al. IncRNA GAS5/miR-223/NAMPT axis modulates the cell proliferation and senescence of endothelial progenitor cells through PI3K/AKT signaling.[J] .J. Cell. Biochem., 2019, 120: 14518-14530.

11. Cui Yigong,Fu Shenglong,Sun Dong et al. EPC-derived exosomes promote osteoclastogenesis through LncRNA-MALAT1.[J] .J. Cell. Mol. Med., 2019, 23: 3843-3854.

12. Quan Hongyu,Liang Mengmeng,Li Nan et al. LncRNA-AK131850 Sponges MiR-93-5p in Newborn and Mature Osteoclasts to Enhance the Secretion of Vascular Endothelial Growth Factor a Promoting Vasculogenesis of Endothelial Progenitor Cells.[J] .Cell. Physiol. Biochem., 2018, 46: 401-417.

13. Jiang Qingfeng,Xing Wenqun,Cheng Jinhua et al. Knockdown of IncRNA XIST Suppresses Cell Tumorigenicity in Human Non-Small Cell Lung Cancer by Regulating miR-142-5p/PAX6 Axis.[J] .Onco Targets Ther, 2020, 13: 4919-4929.

14. Hai Bao,Pan Xiaoyu,Du Chuanchao et al. LncRNA XIST Promotes Growth of Human Chordoma Cells by Regulating miR-124-3p/iASPP Pathway.[J] .Onco Targets Ther, 2020, 13: 4755-4765.

15. Wen Ji-Feng,Jiang Yong-Qing,Li Chao et al. LncRNA-XIST promotes the oxidative stress-induced migration, invasion, and epithelial-to-mesenchymal transition of osteosarcoma cancer cells through miR-153-SNAI1 axis.[J] .Cell Biol. Int., 2020, DOI囚10.1002/cbin.11405.

16. Liu Yunke,Liu Ke,Tang Chao et al. Long non-coding RNA XIST contributes to osteoarthritis progression via miR-149-5p/DNMT3A axis.[J] .Biomed. Pharmacother., 2020, 128: 110349.

17. Li Mingli, Meng Xiangli,Li Mingxuan,MiR-126 promotes esophageal squamous cell carcinoma via inhibition of apoptosis and autophagy.[J] .Aging (Albany NY), 2020, 12: DOI区 10.18632/aging.103379.

18. Sabo Alexandru A,Birolo Giovanni,Naccarati Alessio et al. Small Non-Coding RNA Profiling in Plasma Extracellular Vesicles of Bladder Cancer Patients by Next-Generation Sequencing: Expression Levels of miR-126-3p and piR-5936 Increase with Higher Histologic Grades.[J] .Cancers (Basel), 2020, 12, DOIه10.3390/cancers12061507.

19. Zhang Lin,Yang Hui,Li Wen-Juan et al. LncRNA MALAT1 Promotes OGD-Induced Apoptosis of Brain Microvascular Endothelial Cells by Sponging miR-126 to Repress PI3K/Akt Signaling Pathway.[J] .Neurochem. Res., 2020, DOIه10.1007/s11064-020-03071-6 .

20. Rad Sima Kianpour,Arya Aditya,Karimian Hamed et al. Mechanism involved in insulin resistance via accumulation of $\beta$-amyloid and neurofibrillary tangles: link between type 2 diabetes and Alzheimer's disease.[J] .Drug Des Devel Ther, 2018, 12: 3999-4021.

21. Liu Li Ying,Wang Wei,Zhao Lin Yu et al. Mir-126 inhibits growth of SGC-7901 cells by synergistically targeting the oncogenes PI3KR2 and Crk, and the tumor suppressor PLK2.[J] .Int. J. Oncol., 2014, 45: 1257-65. 
22. Chang Lili,Liang Jinning,Xia Xiujuan et al. miRNA-126 enhances viability, colony formation, and migration of keratinocytes HaCaT cells by regulating PI3 K/AKT signaling pathway.[J] .Cell Biol. Int., 2019, 43: 182-191.

23. Miko E, Margitai Z, Czimmerer Z, et al: miR-126 inhibits proliferation of small cell lung cancer cells by targeting SLC7A5. FEBS Lett 585: 1191-1196, 2011.

24. Yang Nana,Tian Hua,Zhan Enxin et al. Reverse-D-4F improves endothelial progenitor cell function and attenuates LPS-induced acute lung injury.[J] .Respir. Res., 2019, 20: 131.

25. Shih Chun-Ming,Lin Feng-Yen,Yeh Jong-Shiuan et al. Dysfunctional high density lipoprotein failed to rescue the function of oxidized low density lipoprotein-treated endothelial progenitor cells: a novel index for the prediction of HDL functionality.[J] .TransI Res, 2019, 205: 17-32.

26. Wang Chen-Yu,Colognori David,Sunwoo Hongjae et al. PRC1 collaborates with SMCHD1 to fold the X-chromosome and spread Xist RNA between chromosome compartments. [J] .Nat Commun, 2019, 10: 2950.

27. Chen Zhenzhang,Hu Xiao,Wu Yuan et al. Long non-coding RNA XIST promotes the development of esophageal cancer by sponging miR-494 to regulate CDK6 expression.[J] .Biomed. Pharmacother., 2019, 109: 2228-2236.

28. Liu Hua,Deng Haoyu,Zhao Yajie et al. LncRNA XIST/miR-34a axis modulates the cell proliferation and tumor growth of thyroid cancer through MET-PI3K-AKT signaling.[J] .J. Exp. Clin. Cancer Res., 2018, 37: 279.

29. Zhu Jia,Zhang Rui,Yang Dongxiang et al. Knockdown of Long Non-Coding RNA XIST Inhibited Doxorubicin Resistance in Colorectal Cancer by Upregulation of miR-124 and Downregulation of SGK1.[J] .Cell. Physiol. Biochem., 2018, 51: 113-128.

30. Liu Xingxiang,Cui Lin,Hua Dong,Long Noncoding RNA XIST Regulates miR-137-EZH2 Axis to Promote Tumor Metastasis in Colorectal Cancer.[J] .Oncol. Res., 2018, 27: 99-106.

31. Xiao Dong,Cui Xiangyan,Wang Xin,Long noncoding RNA XIST increases the aggressiveness of laryngeal squamous cell carcinoma by regulating miR-124-3p/EZH2.[J] .Exp. Cell Res., 2019, 381: 172-178.

32. Wang Jinglu,Cai Haibo,Dai Zhaoxia et al. Down-regulation of IncRNA XIST inhibits cell proliferation via regulating miR-744/RING1 axis in non-small cell lung cancer.[J] .Clin. Sci., 2019, 133: 1567-1579.

33. Kong Qinglei,Zhang Shaoquan,Liang Caiqian et al. LncRNA XIST functions as a molecular sponge of miR-194-5p to regulate MAPK1 expression in hepatocellular carcinoma cell.[J] .J. Cell. Biochem., 2018, 119: 4458-4468.

34. Lv Gong-Yi,Miao Jun,Zhang Xiao-Lin,Long Noncoding RNA XIST Promotes Osteosarcoma Progression by Targeting Ras-Related Protein RAP2B via miR-320b.[J] .Oncol. Res., 2018, 26: 837846.

35. Zhou Tao,Qin Guowei,Yang Liehong et al. LncRNA XIST regulates myocardial infarction by targeting miR-130a-3p.[J] .J. Cell. Physiol., 2019, 234: 8659-8667. 
36. Li Lei,Lv Guohua,Wang Bing et al. The role of IncRNA XIST/miR-211 axis in modulating the proliferation and apoptosis of osteoarthritis chondrocytes through CXCR4 and MAPK signaling.[J] .Biochem. Biophys. Res. Commun., 2018, 503: 2555-2562.

37. Gu Shixin,Xie Rong,Liu Xiaodong et al. Long Coding RNA XIST Contributes to Neuronal Apoptosis through the Downregulation of AKT Phosphorylation and Is Negatively Regulated by miR-494 in Rat Spinal Cord Injury.[J] .Int J Mol Sci, 2017, 18.

38. Sabo Alexandru A,Birolo Giovanni,Naccarati Alessio et al. Small Non-Coding RNA Profiling in Plasma Extracellular Vesicles of Bladder Cancer Patients by Next-Generation Sequencing: Expression Levels of miR-126-3p and piR-5936 Increase with Higher Histologic Grades.[J] .Cancers (Basel), 2020, 12.

39. Zhou Xiaofeng,He Yingting,Jiang Yao et al. MiR-126-3p inhibits apoptosis and promotes proliferation by targeting phosphatidylinositol 3-kinase regulatory subunit 2 in porcine ovarian granulosa cells.[J] .Asian-australas. J. Anim. Sci., 2020, 33: 879-887.

40. Luo Wenzheng,Yan Dongming,Song Zhenyu et al. miR-126-3p sensitizes glioblastoma cells to temozolomide by inactivating Wnt/ $\beta$-catenin signaling via targeting SOX2.[J] .Life Sci., 2019, 226: 98-106.

41. Liu R,Zhang Y-S,Zhang S et al. MiR-126-3p suppresses the growth, migration and invasion of NSCLC via targeting CCR1.[J] .Eur Rev Med Pharmacol Sci, 2019, 23: 679-689.

42. Yuan Xiaolong,Deng Xi,Zhou Xiaofeng et al. MiR-126-3p promotes the cell proliferation and inhibits the cell apoptosis by targeting TSC1 in the porcine granulosa cells.[J] .In Vitro Cell. Dev. Biol. Anim., 2018, 54: 715-724.

43. Ohta Momoka,Kihara Toshie,Toriuchi Kohki et al. IL-6 promotes cell adhesion in human endothelial cells via microRNA-126-3p suppression.[J] .Exp. Cell Res., 2020.DOI:10.1016/j.yexcr.2020.112094.

44. Xue Wen-Long,Chen Rui-Qin,Zhang Qing-Qing et al. Hydrogen sulfide rescues high glucose-induced migration dysfunction in HUVECs by upregulating miR-126-3p.[J] .Am. J. Physiol., Cell Physiol., 2020, 318: C857-C869.

45. Pan Jiaji,Qu Meijie,Li Yongfang et al. MicroRNA-126-3p/-5p Overexpression Attenuates Blood-Brain Barrier Disruption in a Mouse Model of Middle Cerebral Artery Occlusion.[J] .Stroke, 2020, 51: 619627.

46. Fu Xi,Niu Tiesheng,Li Xiaodong,MicroRNA-126-3p Attenuates Intracerebral Hemorrhage-Induced Blood-Brain Barrier Disruption by Regulating VCAM-1 Expression.[J] .Front Neurosci, 2019, 13: 866.

47. Cheng Zhihua,Luo Cong,Guo Zhilin,LncRNA-XIST/microRNA-126 sponge mediates cell proliferation and glucose metabolism through the IRS1/PI3K/Akt pathway in glioma.[J] .J. Cell. Biochem., 2020, 121: 2170-2183.

48. Ma S, Charron J, Erikson RL. Role of Plk2 (Snk) in mouse development and cell proliferation. Mol Cell Biol. 2003,23(19):6936-6943.

49. Xia Xiangping,Cao Fang,Yuan Xiaolu et al. Low expression or hypermethylation of PLK2 might predict favorable prognosis for patients with glioblastoma multiforme.[J] .PeerJ, 2019, 7: e7974. 
50. Li Wenhu,Zhang Xianliao,Xi Xinhua et al. PLK2 modulation of enriched TAp73 affects osteogenic differentiation and prognosis in human osteosarcoma.[J] .Cancer Med, 2020, 9: 4371-4385.

51. Matthew Elizabeth M,Yang Zhaohai,Peri Suraj et al. Plk2 Loss Commonly Occurs in Colorectal Carcinomas but not Adenomas: Relationship to mTOR Signaling.[J] .Neoplasia, 2018, 20: 244-255.

52. Liu Wenjing,Zha Zhuqing,Wang Haitao,Upregulation of microRNA-27a inhibits synovial angiogenesis and chondrocyte apoptosis in knee osteoarthritis rats through the inhibition of PLK2.[J] .J. Cell. Physiol., 2019, 234: 22972-22984.

53. Zhao Duo,Shun Edward,Ling Fengjun et al. Plk2 Regulated by miR-128 Induces Ischemia-Reperfusion Injury in Cardiac Cells.[J] .Mol Ther Nucleic Acids, 2020, 19: 458-467.

\section{Figures}

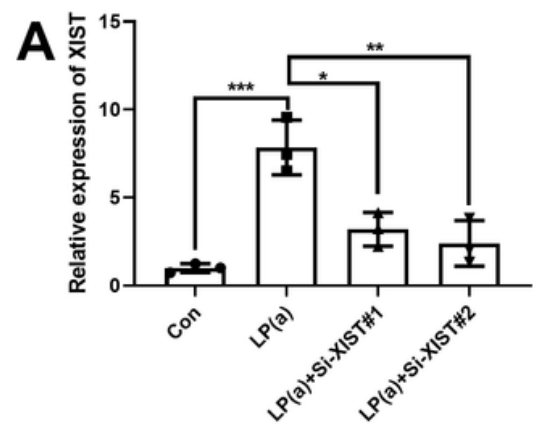

B
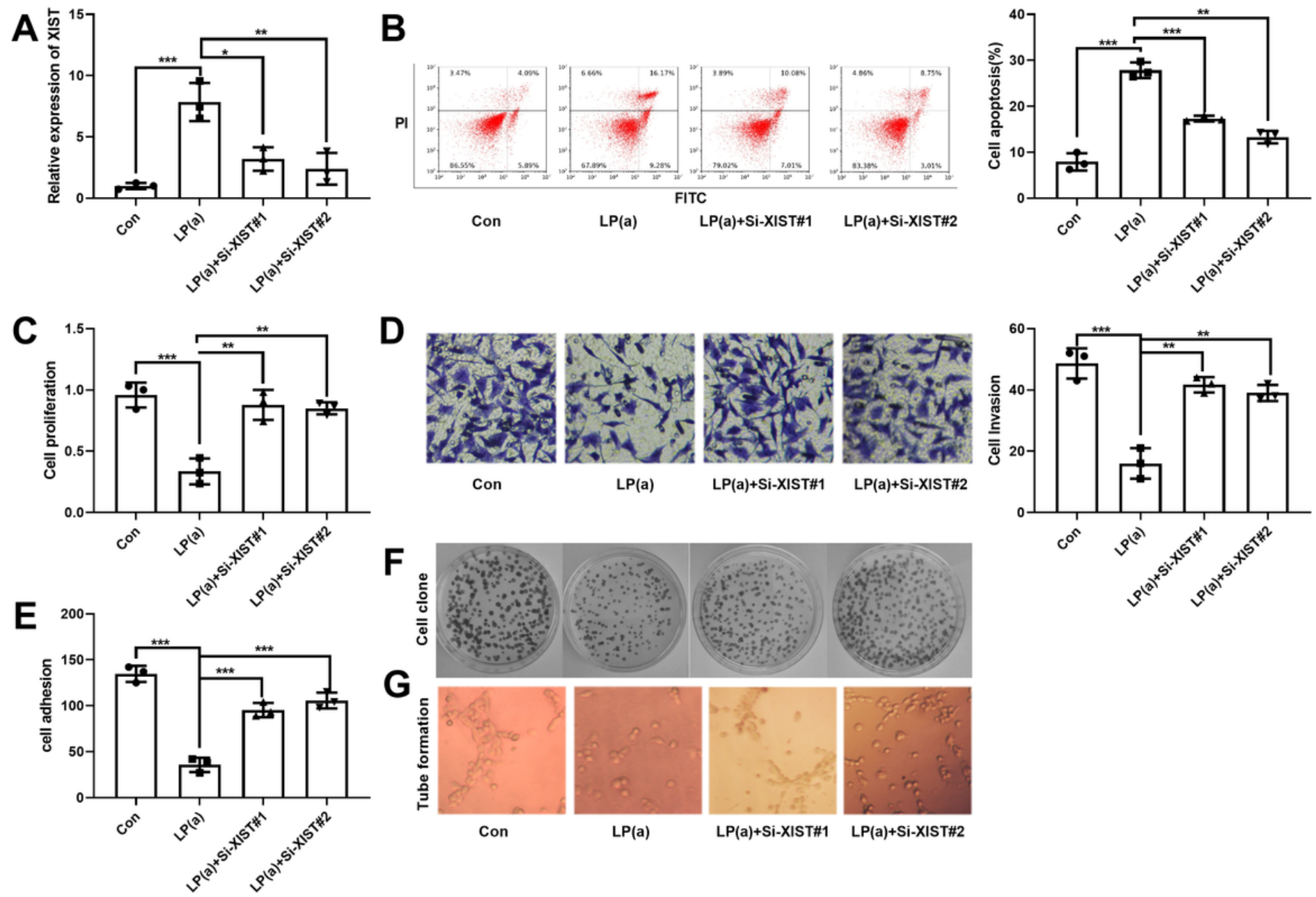

D
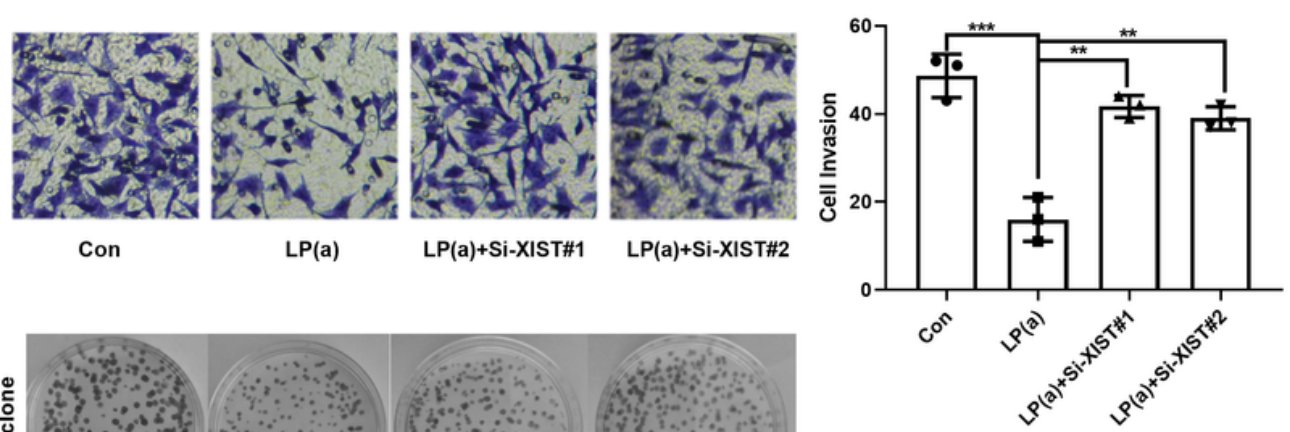

Figure 1

Knocking down IncRNA XIST significantly reduced the injury of LP(a)-mediated EPCs A PCR was carried out to test the effect of knocking down XIST in EPCs, B-C EPCs were treated with LP(a) at a dose of 100 $\mathrm{ng} / \mathrm{mL}$ for 6 hours, and EPC apoptosis was verified by flow cytometry (B), while cell proliferation was 
examined by MTT (C), D Transwell assay was performed to determine cell migration. E Cell adhesion assay was conducted to monitor the changes in cell adhesion. F Colony formation assay was implemented to examine the colony formation of EPCs, G Angiogenesis assay detected the angiogenesis

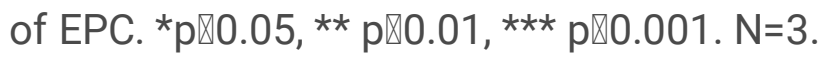
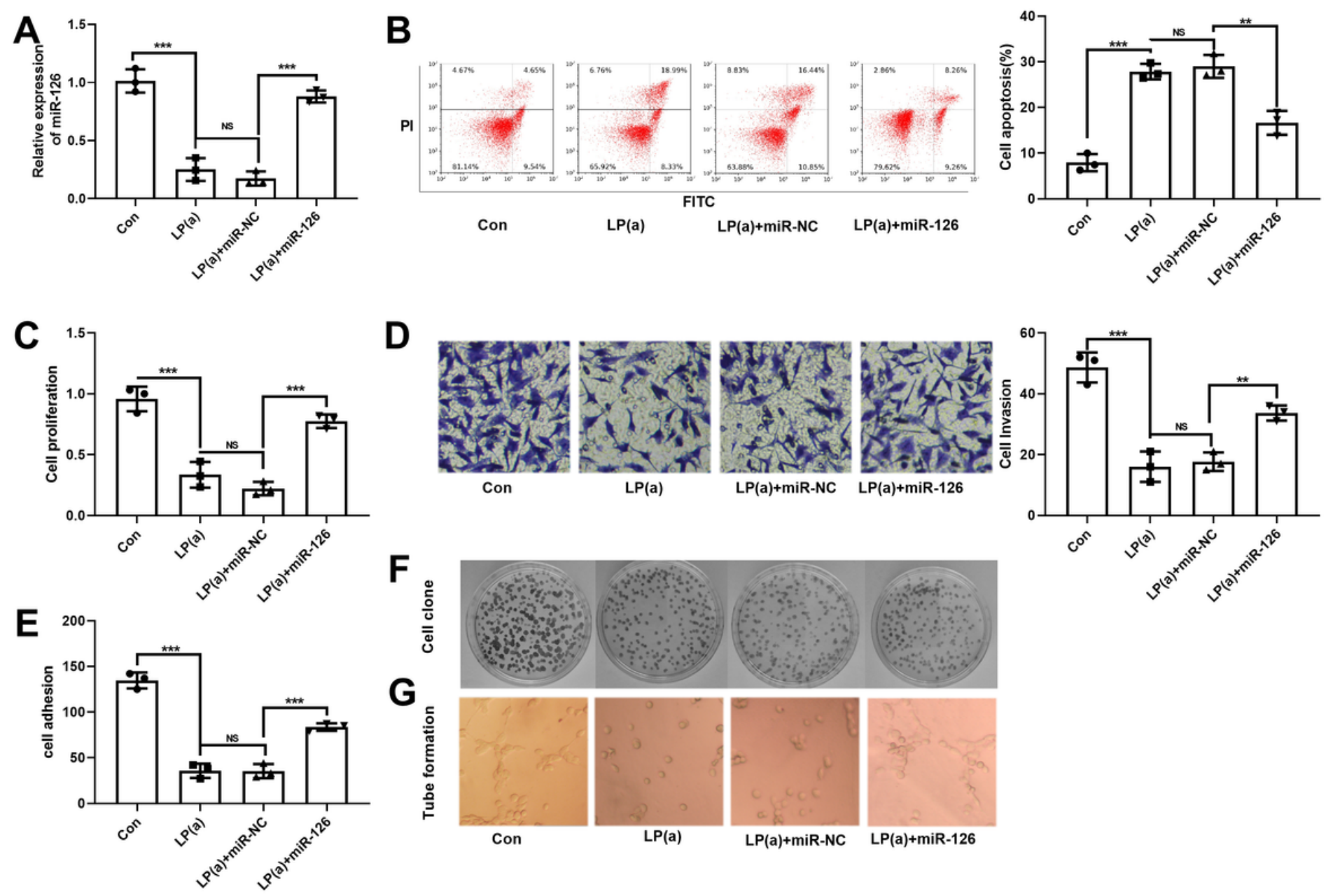

Figure 2

Overexpressing miR-126 notably mitigated the injury of LP(a)-mediated EPCs A The effect of miR-126 overexpression in EPCs was determined by PCR, B-C EPCs were treated with LP(a) at a dose of 100 $\mathrm{ng} / \mathrm{mL}$ for 6 hours. Apoptosis was examined by flow cytometry (B), and cell proliferation was testified by MTT (C), D Cell migration was monitored by Transwell assay. E Adhesion assay was implemented to monitor the changes in cell adhesion. F Colony formation experiment was carried out to verify the colony formation of EPCs, G Angiogenesis assay was employed to determine EPC angiogenesis. ${ }^{\star} \mathrm{P}<0.05$, ${ }^{\star *} \mathrm{P}<$ $0.01, * \star * \mathrm{P}<0.001 . \mathrm{N}=3$. 
A

B

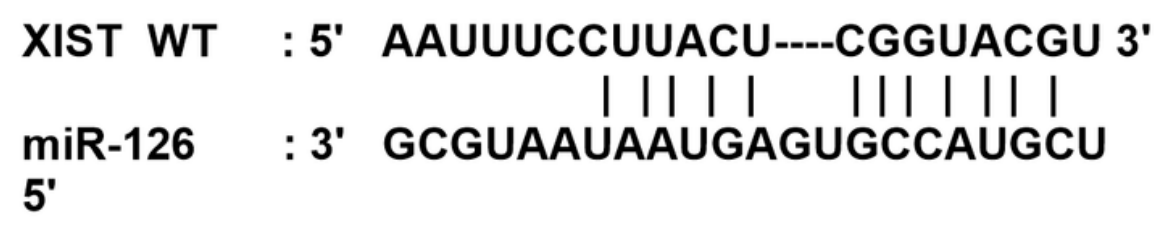

XIST MUT : 5' AAUUUCCAAGUC----GgUACCGU 3'

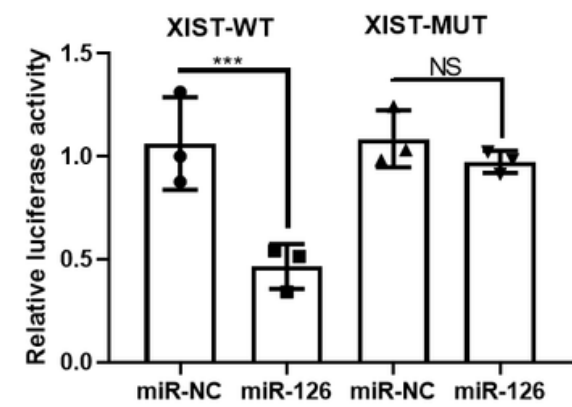

c
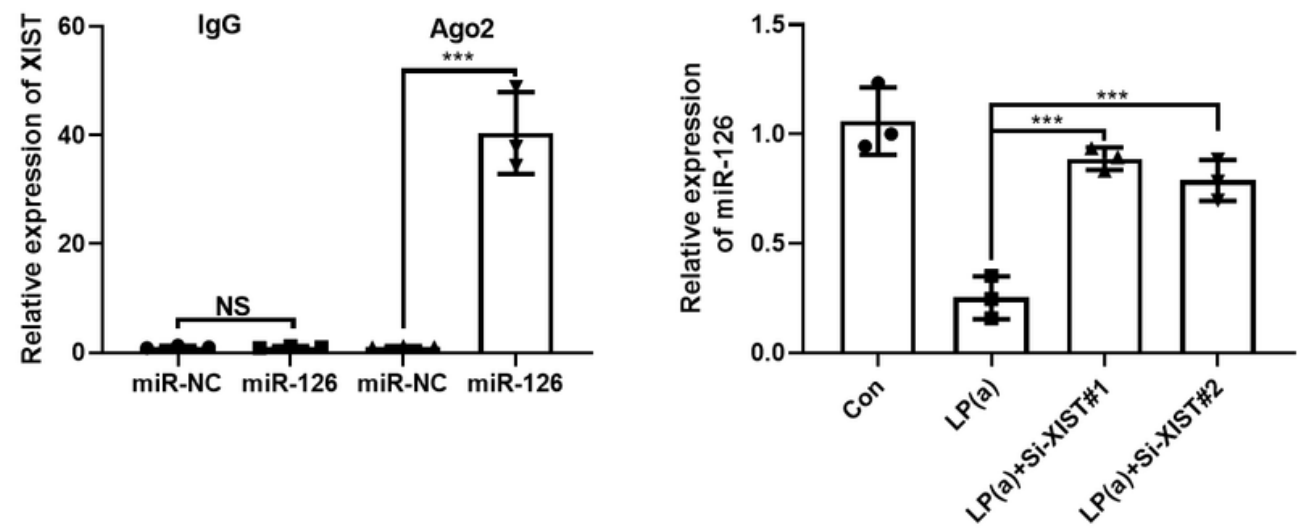

Figure 3

LncRNA XIST targeted miR-126 A The association between XIST and miR-126 was determined by the Starbase, B-C Dual-luciferase reporter assay (B) and RIP experiment (C) confirmed the targeted relationship between XIST and miR-126-3p, D PCR was employed to verify the changes of miR-126. *p》 $0.05, \star \star \mathrm{p} \otimes 0.01, \star \star \star \mathrm{p} \otimes 0.001 . \mathrm{N}=3$. 

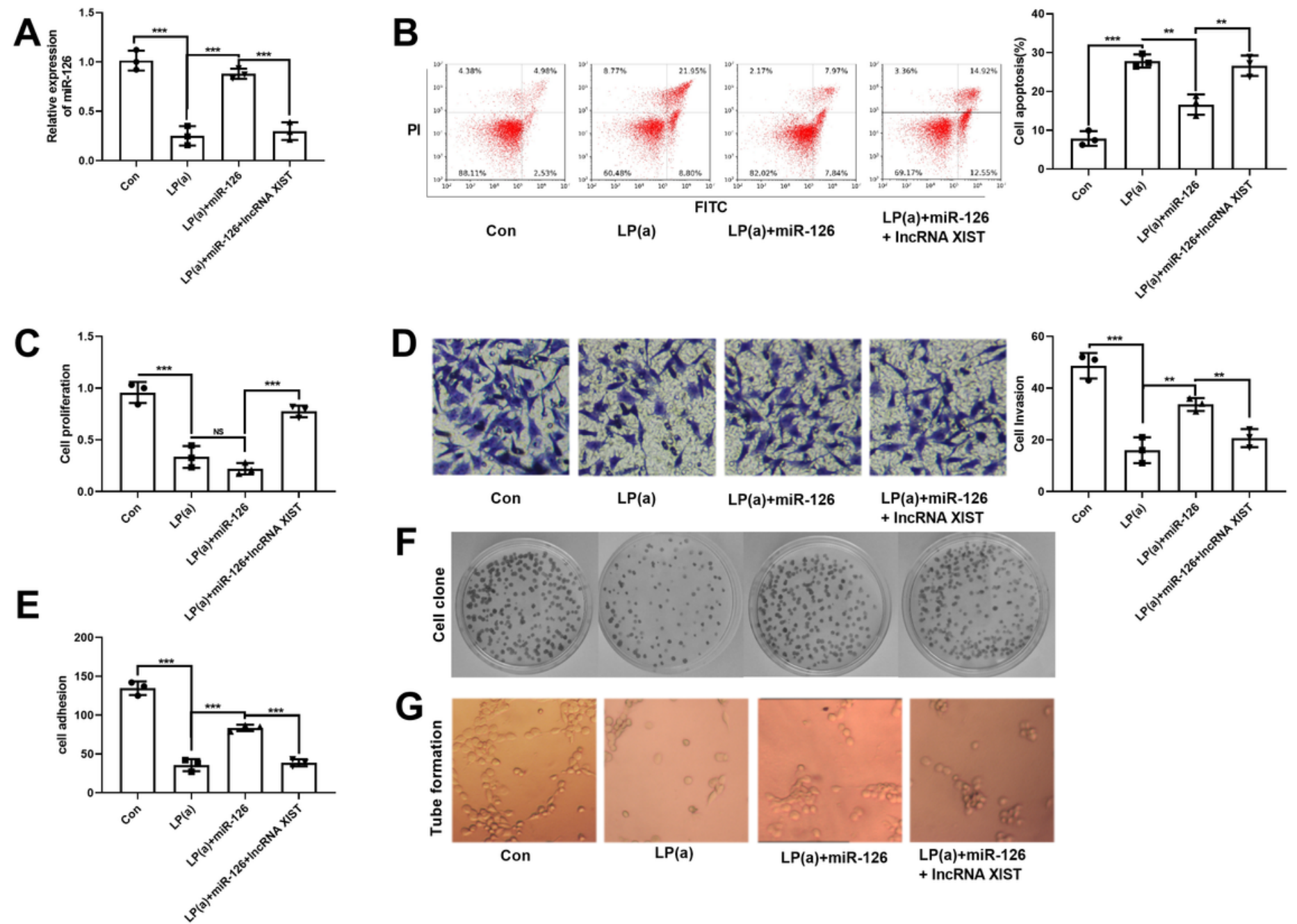

\section{Figure 4}

IncRNA XIST targeted miR-126 to modulate the injury of LP(a)-treated EPCs A PCR was employed to verify the changes of miR-126 after supplementing XIST overexpressed plasmid in EPCs overexpressing miR-126. B-C LP(a) at a dose of $100 \mathrm{ng} / \mathrm{mL}$ was applied to EPCs for 6 hours after overexpressing miR126 and adding XIST overexpression plasmid. EPC apoptosis was monitored by flow cytometry (B), and cell proliferation was determined by MTT (C), D Cell migration was detected by Transwell. E Cell adhesion assay was employed to monitor the change of cell adhesion ability. F Colony formation experiment was implemented to determine the colony formation of EPCs, G angiogenesis assay was adopted to verify

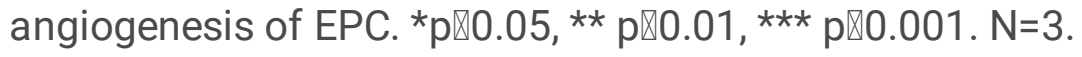




\section{PLK2 WT : 5' AgAUggacAUAUguggugguacGA 3' | : : | || | || \\ miR-126 : 3' GCGUAAUAAUGAGUGCCAUGCU 5' \\ PLK2 MUT: 5' AGAUgGACAUAUGAggGUAGUCGA 3'}

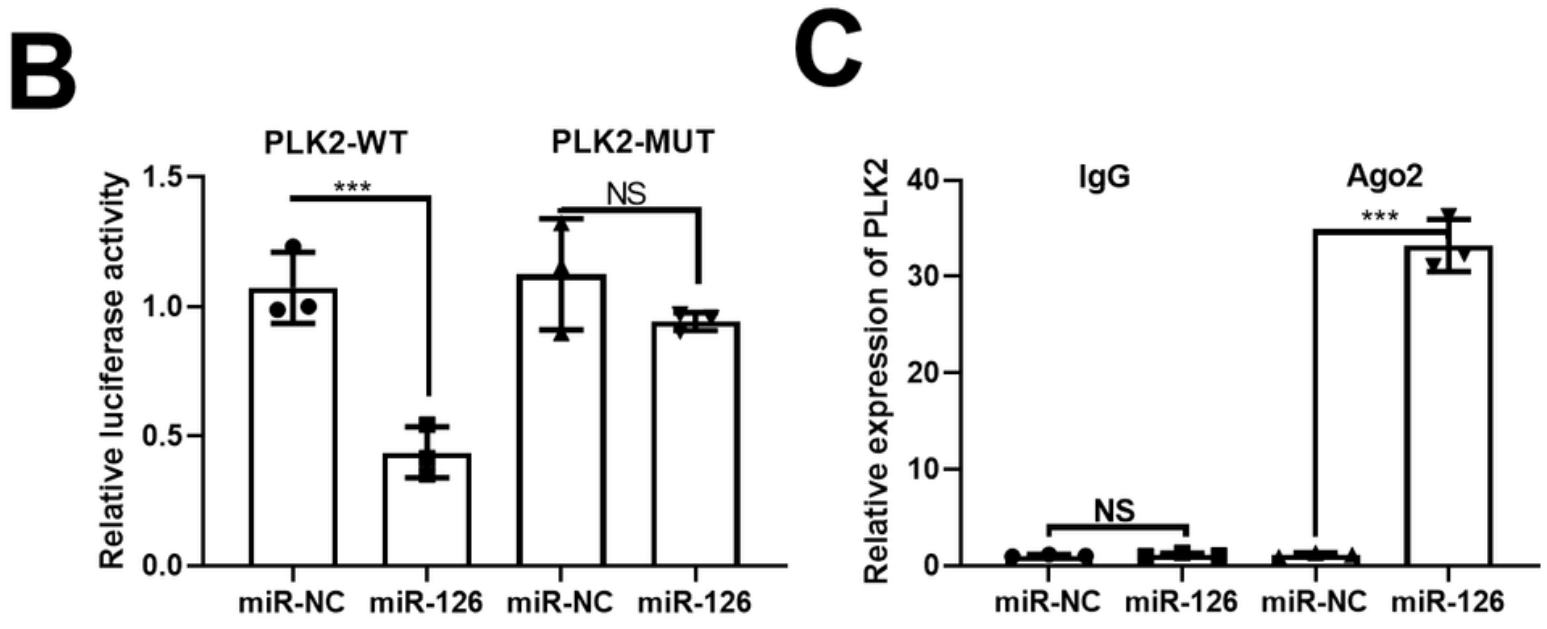

Figure 5

miR-126 targeted PLK2 A The Starbase was adopted to clarify the association between PLK2 and miR126, B-C Dual-luciferase reporter assay (B) and RIP experiment (C) were implemented to determine the

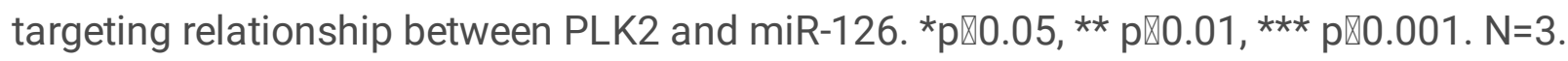



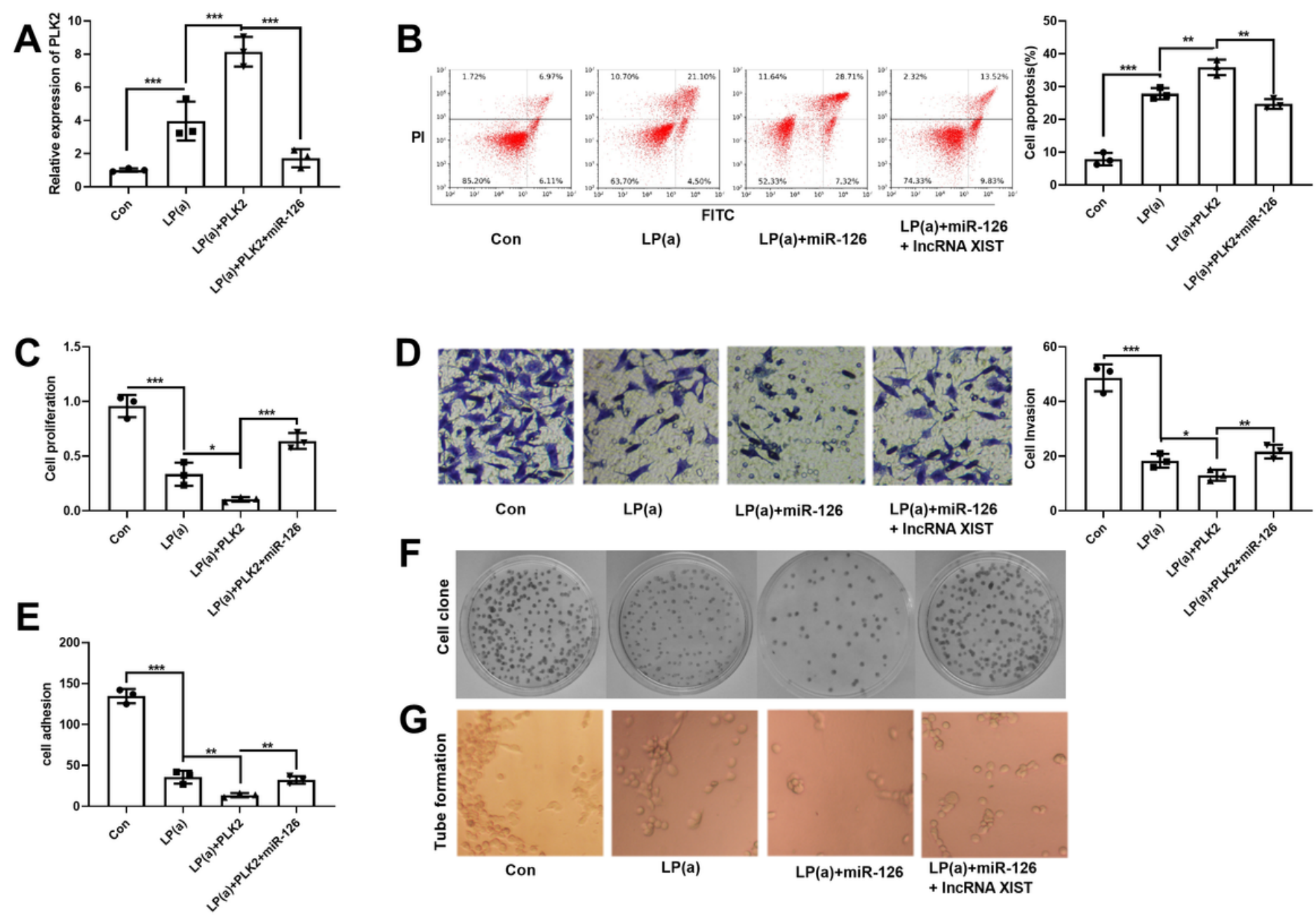

\section{Figure 6}

miR-126 targeted PLK2 to regulate injury of LP(a)-mediated EPCs A Changes of PLK2 after supplementing miR-126 mimics in EPCs were monitored by RT-PCR and WB. B-C LP(a) at a dose of 100 $\mathrm{ng} / \mathrm{mL}$ was applied to EPCs overexpressing PLK2 for 6 hours after supplementing with miR-126 mimics. EPC apoptosis was verified by flow cytometry (B), and cell proliferation was determined by MTT (C), D Cell migration was examined by Transwell. E Cell adhesion assay was used to detect the change of cell adhesion. F Colony formation assay was implemented to test the colony formation of EPCs, $G$

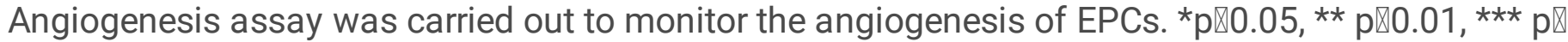
0.001.N=3. 